\title{
QUASI-FREE SCATTERING OF POLARIZED ELECTRONS BY POLARIZED NUCLEAR PROTONS *
}

\author{
V.E. HERSCOVITZ*, Th.A.J. MARIS* and M.R. TEODORO \\ Instituto de Física, Universidade Federal do Rio Grande do Sul, 90000 Porto Alegre, RS, Brasll
}

Received 4 January 1977

Revised manuscript received 1 April 1977

\begin{abstract}
It is shown that quast-free electron scattering in a suitable geometry takes place on strongly polarized nuclear protons. A calculation for the single particle model of ${ }^{16} \mathrm{O}$ predicts large asymmetries for the scattering of polarized electrons.
\end{abstract}

Soon after the first quasi-free $(p, 2 p)$ experiments were performed, it was recognized that in these processes the incoming proton scatters on a nuclear proton which is in general polarized [1] by the nuclear spin-orbit force. In an asymmetrical situation this polarization does not average out, because the multiple scattering of the emerging proton parr suppresses the contributions of certain regions of the nucleus to the quasi-free cross section.

Recently it has been theoretically [2,3] and experimentally [4] shown that one may measure the effective polarization in quasi-free scattering with the help of polarized incoming protons, making use of the large difference of the cross sections for scattering of medium energy free protons with parallel and opposite spins. The first experimental results [4] agree well with the theoretical expectations.

It is the purpose of this letter to show in a simple but rather realistic model that the polarization of the nuclear protons may also give interestıng effects in $\left(e, e^{\prime} p\right)$ quasi-free scattering. We discuss as an example the kinematical situation sketched in fig. 1, which is convenient from a theoretical point of view, although experimentalists may judge differently.

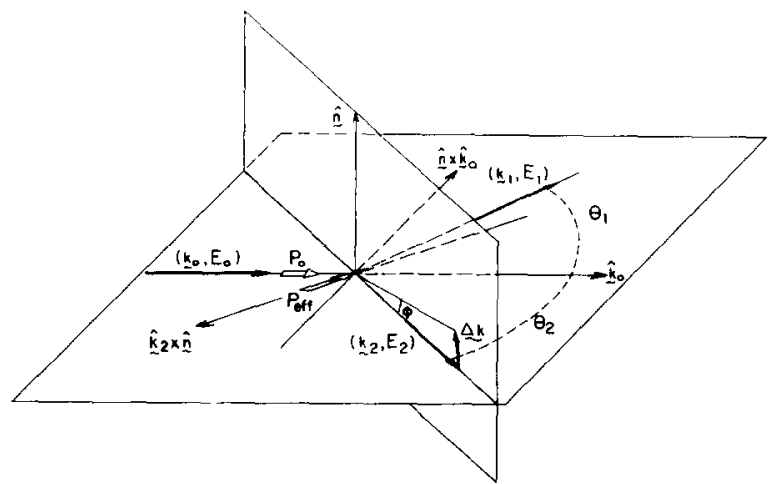

Fig. 1 The geometry of the proposed experiment.

In fig. $1\left(k_{0}, E_{0}\right),\left(k_{1}, E_{1}\right)$ and $\left(k_{2}, E_{2}\right)$ denote the momenta and energies of the incoming and emerging electrons and of the emerging proton, respectively. Initially, the momenta are chosen such that $k_{0}=k_{1}+k_{2}$, and one is therefore essentially scattering on nuclear protons in rest. For the nucleus we chose ${ }^{16} \mathrm{O}$, considered in the ex-

\footnotetext{
* Work partially supported by CNPq (Brasil), FINEP (Brasil) and GTZ (Germany

* Research Fellow, CNPq (Brasil).
} 
treme single particle model, and the energies $E_{i}$ are so selected that one is observing protons knocked-out of ether the $p_{1 / 2}$ or the $p_{3 / 2}$ subshell with separation energies of 12 or $18 \mathrm{MeV}$, respectively; for the kinematics we took an average separation energy of $15 \mathrm{MeV}$. We vary the direction of the proton momentum, moving it through an angle $\phi$ in a plane orthogonal to the plane of the electron momenta; $\boldsymbol{k}_{0}, \boldsymbol{k}_{1}$ and $\left|\boldsymbol{k}_{2}\right|$ are kept fixed. In this way the original momentum of the outgoing proton obtains an addition $\Delta \boldsymbol{k}$ and one is now observing nuclear protons having approxımately momentum $\Delta \boldsymbol{k}$. By varying $\phi$ one is thus able to measure a distribution of nuclear proton momenta which are about orthogonal to the electron plane.

A simple physical consideration $[2,5]$ suggests that the knocked out proton was effectively polarized in the nucleus. Formally this polarization is given by [1]:

$$
\boldsymbol{P}_{\mathrm{eff}}=\frac{2 \operatorname{Re}\left(\sum_{m} g_{m}^{(+)} g_{m}^{(-)}\right) ; 2 \operatorname{Im}\left(\sum_{m} g_{m}^{(+)} g_{m}^{((-)}\right) ; \sum_{m}\left(\left|g_{m}^{(+)}\right|^{2}-\left|g_{m}^{\prime(-)}\right|^{2}\right)}{\sum_{m} \sum_{n}\left|g_{m}^{((n)}\right|^{2}}
$$

As indicated in fig. 1, $\boldsymbol{P}_{\text {eff }}$ is orthogonal to the plane in which we vary the proton momentum, because this is a plane of mirror symmetry for the calculation of the effective polarization, the electron only entering by its energy and momentum transfer which is in that plane. In formula (1), $g_{m}^{( \pm)}$represent the two spin components of the distorted momentum amplitudes $g_{m}^{\prime}$; the quantization axis is taken in the direction $\hat{k}_{2} \times \hat{n}$, where $\hat{n}$ is the unit vector parallel to $\boldsymbol{k}_{0} \times \boldsymbol{k}_{1}$.

We have calculated expression (1) in the WKB-approxımation [1], using the following parameters. Energy of emerging proton: $84 \mathrm{MeV}$. Square well nuclear wavefunctions with correct binding energies. Square well optical proton potential: $V=(-32.9-\mathrm{i} 13.0) \mathrm{MeV}$. Radii of both square wells: $3.41 \mathrm{fm}$.

The resulting effective polarization and distorted momentum distribution for the $\mathrm{p}_{1 / 2}$ states are given in figs. $2 a, b$. The effective polarization at the maxima of the momentum distribution is about 80 percent! The real part of the optical potential is important, as it is in the $(p, 2 p)$ case $[2,6]$; without it the polarization turns out to be about half as large. The maxima of the distorted momentum distribution are 0.68 times the ones of the undistorted distribution, in agreement with earlier calculations. The value of $P_{\text {eff }}$ for the $\mathrm{p}_{3 / 2}$ states is half as large and of opposite sign, because there are twice as many particles in this subshell and the effect averages out for the complete p-shell.

The expression in the DWIA of the quasi-free cross section for the present geometry is in the usual notation [7]

$$
\int \frac{\mathrm{d} \sigma}{\mathrm{d} \Omega_{1} \mathrm{~d} E_{1} \mathrm{~d} \Omega_{2} \mathrm{~d} E_{2}} \mathrm{~d} E_{\mathrm{S}}=\left(\frac{\mathrm{d} \sigma}{\mathrm{d} \Omega_{1}}\right)^{\text {free }} \frac{k_{2}}{\hbar^{2} c^{2}}\left(M_{\mathrm{p}} c^{2}+2 E_{0} \sin ^{2}\left(\frac{\theta}{2}\right)\right) \sum_{m} \sum_{n}\left|g_{m}^{\prime(n)}\left(\boldsymbol{k}_{1}+\boldsymbol{k}_{2}-\boldsymbol{k}_{0}\right)\right|^{2} .
$$

$\left(\mathrm{d} \sigma / \mathrm{d} \Omega_{1}\right)^{\text {free }}$ is in our case the cross section for elastıc scattering of electrons on protons with the polarization $\boldsymbol{P}_{\text {eff }}$ of eq. (1), orthogonal to the proton momentum and parallel to the scattering plane; a sum over final spins has been performed. Because of the special geometry, the dependence [7] on the angle $\phi$, between the proton momentum and the electron plane, is to a good approximation only contained in $\Sigma\left|g^{\prime}\right|^{2}$ and in $P_{\text {eff }}$. One has [8] for incoming electrons with polarization $\boldsymbol{P}_{0}$ suffering a four-momentum transfer $q$ :

$$
\left(\frac{\mathrm{d} \sigma}{\mathrm{d} \Omega}\right)^{\text {free }}=\left(\frac{\mathrm{d}}{\mathrm{d} \Omega}\right)_{0}\left[1+\left(\boldsymbol{P}_{0} \cdot \hat{\boldsymbol{k}}_{0}\right)\left(\boldsymbol{P}_{\mathrm{eff}} \cdot \hat{\boldsymbol{k}}_{0}\right) M_{11}+\left(\boldsymbol{P}_{0} \cdot \hat{\boldsymbol{k}}_{0}\right)\left(\boldsymbol{P}_{\mathrm{eff}} \cdot\left(\hat{\boldsymbol{n}} \times \hat{\boldsymbol{k}}_{0}\right)\right) M_{13}\right]
$$

where

$$
\left(\frac{\mathrm{d} \sigma}{\mathrm{d} \Omega}\right)_{0}=\left(\frac{e^{2}}{4 \pi}\right)^{2}(\hbar c)^{2} \frac{\left[1+\eta \mu^{2}+2(1+\mu)^{2} \operatorname{tg}^{2}\left(\frac{\theta_{1}}{2}\right)\right]\left|F_{1}\right|^{2} \cos ^{2}\left(\frac{\theta_{1}}{2}\right)}{4 M_{\mathrm{p}}^{2} c^{4} \xi^{2}\left(1+2 \xi \sin ^{2}\left(\frac{{ }_{1}}{2}\right)\right) \sin ^{4}\left(\frac{{ }^{\theta}}{2}\right)}, \quad M_{11}=\left[\eta\left(1-\mu+\xi^{-1}\right)-\xi\right] \rho \operatorname{tg}\left(\frac{1}{2}\right)
$$




$$
\begin{aligned}
& M_{13}=\rho \eta\left(\xi^{-1}-\mu\right), \quad \rho=\frac{2(1+\mu) \operatorname{tg}\left(\theta_{1} / 2\right)}{1+\eta \mu^{2}+2 \eta(1+\mu)^{2} \operatorname{tg}^{2}\left(\theta_{1} / 2\right)} ; \quad \xi=\frac{E_{0}}{M_{\mathrm{p}} c^{2}} ; \quad \eta=\frac{\xi^{2} \sin ^{2}\left(\theta_{1} / 2\right)}{1+2 \xi \sin ^{2}\left(\theta_{1} / 2\right)} \\
& \mu(\eta)=\frac{F_{2}\left(q^{2}\right)}{F_{1}\left(q^{2}\right)}
\end{aligned}
$$

From eq. (3) follows that the proton polarization can be observed with (longitudinally) polarized electrons. As an example we consider $500 \mathrm{MeV}$ incoming electrons, scattered through an angle of $57^{\circ}$, resulting in $84 \mathrm{MeV}$ protons emerging under an angle of $50^{\circ}$. Using experimental proton form factors [9], one finds:

$$
M_{11}=-0.533, \quad M_{13}=0.035 \text {. }
$$

The combination of eqs. (1)-(4) gives the quasi-free cross sections for incoming longitudinally polarized electrons. These are shown for the $\mathrm{p}_{1 / 2}$ states in fig. $2 \mathrm{c}$, in dependence of the angle $\phi$. The $\mathrm{p}_{3 / 2}$ states give an opposite and half as large asymmetry effect. Calculations for other medium electron energies and (suitable) scattering angles give results of quite comparable size.

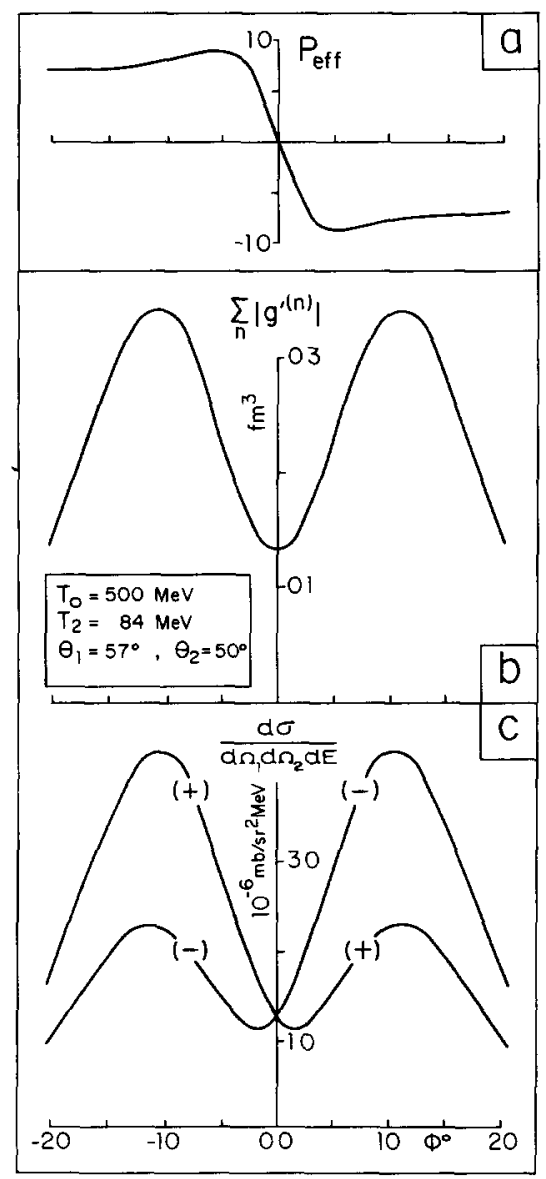

Fig. 2 (a) The effective polarization of the nuclear proton in the $p_{1 / 2}$ subshell of ${ }^{16} \mathrm{O}$ in dependence of the angle $\phi$ between the proton momentum and the electron plane. (b) The distorted momentum distribution. (c) The quasi-free cross section for electrons, longitudinally polarized in the durection of $(+)$ and opposite to $(-)$ the beam momentum. 
The predicted large polarization effects are determined by the assumed models of the reaction and nucleus. A comparison with future experiments would constitute a significant test of the generally assumed quasi-free reaction mechanism and sufficiently accurate measurements would lead to detailed information on the distortion of the proton wave as well as on the nuclear states involved. It would also be interesting to see a new case in which nuclear protons function as a naturally polarized target and this might suggest other applications.

We make some final remarks:

(a) Mirroring the process of fig. 1 through the electron plane, one sees that the curves $(+)$ and $(-)$ in fig. $2 \mathrm{c}$ are each other mirror images (up to the accuracy of parity [10] conservation).

(b) We have estimated the depolarization of the incoming electron in the nuclear Coulomb field by a WKB approximation of the relevant Dirac equation. For ${ }^{16} \mathrm{O}$ this turns out to be an effect of a few percent and we have neglected it. But already for a nucleus like ${ }^{40} \mathrm{Ca}$ the depolarization becomes appreciable and should probably be taken into account.

(c) The spin-orbit coupling of the outgoing proton has been neglected. If one would measure the final proton spins, the resulting error could be serious. However, because we sum over final proton spins, the error is expected to be small; the spin-orbit distortion mainly rotates the spin of the proton, approximately acting as a unitary operator in spin space, which has no effect after spin summation, because of conservation of probability.

(d) We have neglected the possibility that the final proton results from a (e, e'n) reaction followed by a charge exchange, because this process is unlikely to result in the final one-hole state of interest. In a pure single particle model and with a single charge exchange, this would demand that the neutron be knocked out of one particular state and then exchange with the proton in the analogue state.

(e) We have neglected off-energy shell effects in the $e-p$ matrix element. Because in the chosen geometry the nuclear target proton moves almost orthogonally to the electron plane, this effect cancels essentially out in the relative cross sections.

We thank C. Schneider for discussions.

\section{References}

[1] Th.A.J. Marıs, Nucl. Phys. 9 (1958/59) 577.

[2] G. Jacob, Th.A.J Mar1s, C. Schneidet and M.R. Teodoro, Phys. Lett. 45B (1973) 181, and Nucl. Phys. A257 (1976) 517

[3] M.R. Teodoro, Doctoral thesis, Universidade Federal do Rio Grande do Sul, 1976, unpublished.

[4] P. Kitching et al., Observation of $J$-dependent effects in quasi-free scattering of polarized protons, TRIUMF preprint (1976).

[5] H.C. Newns, Proc. Phys Soc. A66 (1953) 477.

[6] Private communication by C.A Miller.

[7] G. Jacob and Th.A.J. Marıs, Nucl. Phys. 31 (1962) 139.

[8] A.I. Akhiezer, L.N. Rosentsveig and I.M. Shmushkevich, Soviet Phys. JETP 6 (1958) 588.

[9] G. Weber, in: 3rd Intern. Symp. on Electron and proton interactions at high energies, Stantord, 1967, p. 59.

[10] P. Winternitz, in: Intern. Conf. on polarızed targets, Berkeley, 1971, p. 145. 